\title{
BF-theory in graphene: a route toward topological quantum computing?
}

\author{
A. Marzuoli ${ }^{\dagger},,(1)$ and G. Palumbo ${ }^{\ddagger},,(2)$ \\ $\dagger$ Dipartimento di Matematica 'F. Casorati', Università degli Studi di Pavia, \\ via Ferrata 1, 27100 Pavia (Italy) \\ † Dipartimento di Fisica Nucleare e Teorica, Università degli Studi di Pavia, \\ via A. Bassi 6, 27100 Pavia (Italy) \\ $\S$ Istituto Nazionale di Fisica Nucleare, Sezione di Pavia, \\ via A. Bassi 6, 27100 Pavia (Italy) \\ (1) E-mail: annalisa.marzuoli@pv.infn.it \\ (2) E-mail: giandomenico.palumbo@pv.infn.it
}

\begin{abstract}
Besides the plenty of applications of graphene allotropes in condensed matter and nanotechnology, we argue that graphene sheets might be engineered to support room-temperature topological quantum processing of information. The argument is based on the possibility of modelling the monolayer graphene effective action by means of a 3d Topological Quantum Field Theory of BF-type able to sustain non-Abelian anyon dynamics. This feature is the basic requirement of recently proposed theoretical frameworks for fault-tolerant and decoherence protected quantum computation.
\end{abstract}

PACS: 03.67.-a, 71.10.-w; 11.15.-q 


\section{Introduction}

The issue of connections between topological quantum field theories (TQFT) in three spacetime dimensions [1, 2] and condensed matter systems in $d=2,3$ has been intensively investigated over the years in a variety of different contexts. The occurrence of topological phases of matter in ground states and the existence of quasi-particle excitations associated with fractionary statistics [3] are the crucial features of many-body microscopic systems sharing topological effective actions. Such collective behaviors have been observed in the Fractional Quantum Hall Effect, cold atoms in optical lattices, Topological Insulators and graphene sheets [4, 5, 6]. Most recent proposals of experimental settings have also fostered theoretical research aimed to classify classes of models supporting topological phases [7, 8].

Topological (or anyonic) quantum computing is a promising territory where models and tools from topological (effective) quantum field theory might find new exciting applications [9, 10]. The basic ingredients of any anyonic-type computation involve: i) the choice of a finite set particle types, i.e. labels specify the possible values of the charges; ii) the assumption that particles can fuse and split according to a set of rules that give the charge of a composite particle in term of the constituents; iii) the assumption that particles trajectories are braided according to rules specifying how pairs (or bipartite subsystems) behave under exchange. 3d TQFT of the Schwarztype [11, 12], as well as 2d (boundary) Conformal Field Theories and charge sectors of families of $(2+1)$-dimensional gauge theories (with finite or compact Lie gauge group) are theoretical frameworks able to support multidimensional unitary representation of the braid group obeying fractionary statistics. However, the requirements of fault-tolerance and stability under local quantum perturbations - achieved when every unitary gate can be approximated within any precision by braiding and fusing transformationsselects more restrictive classes of theories. For instance [13], doubled nonAbelian Chern-Simons (CS) theories supporting non-Abelian statistics, provide such a PT-invariant theoretical framework. This circumstance provides the basic motivation of this letter since any $3 \mathrm{~d}$ doubled Chern-Simons TQFT can be converted into a BF-theory, a fact that has been already exploited in a number of recent approaches to infrared regimes of many-body quantum systems [14, 15, 16, 17, 18].

The goal of this work is to exploit the doubled CS $\leftrightarrow$ BF correspondence in order to provide theoretical evidence for the emergence of non-Abelian 
anyons in monolayer graphene, thus opening the challenging opportunity for a 'room temperature' topological quantum computer. In the present context non-Abelian anyons are induced by some amount of disorder due to random impurities and by resorting to the replica method it is shown that the effective action of the system is given by a non-Abelian BF-type action with $U(2)$ playing the role of the gauge group (for any choice of the level $\kappa>0$ ). The two emergent basic fields $\mathbf{A}$ and $\mathbf{B}$, the gauge potential (connection) and the so-called $B$-field to be associated with the presence of vortices, turn out to be related in a natural way to their microscopic counterparts. Finally, the issue of (gauge invariant) quantum observables to be associated with the effective theory is briefly addressed.

It is worth stressing that, unlike pure $\mathrm{CS}$, the BF framework -originally formulated in terms of continuous geometric structures and as such used in the following- possesses natural discretized counterparts given by the class of the Turaev-Viro state sum models ( $c f r$. a few more remarks in the last section). The issue of giving an equivalent lattice formulation of the effective $\mathrm{BF}$ action for graphene is crucial for assessing and improving the potentialities of the model in view of realistic applications for quantum computational purposes. Progress in this direction is under study and will be presented elsewhere.

\section{Graphene: microscopic description}

Graphene [19] is a two-dimensional planar honeycomb lattice with strongly bounded carbon atoms placed at the sites, and the physical properties of this material are under intense scrutiny [20, 21].

At room temperature, near the Fermi points, the charge carries exhibit a relativistic behavior and thus their wave functions are suitably described by a $(2+1)$-dimensional massless Dirac equation [22]. This property is related to the fact that a vanishing gap between the conductance and valence bands in the energy spectrum has been experimentally measured.

The massless Dirac equation is explicitly given by

$$
i \gamma^{\mu} \partial_{\mu} \psi=0
$$


where $\mu=0,1,2$ and the bi-spinor is

$$
\psi=\left(\begin{array}{c}
\psi_{A}^{+} \\
\psi_{B}^{+} \\
\psi_{A}^{-} \\
\psi_{B}^{-}
\end{array}\right)
$$

where $A$ and $B$ are labels denoting the two triangular sublattices of the honeycomb lattice and + and - refer to the two distinct Fermi points. Both types of indices represent internal degrees of freedom of the charge carriers in graphene. Consequently the $\gamma^{\mu}$ are $4 \times 4$ Dirac matrices and in the following the chiral representation will be always used.

It is possible to get a gap in several different manners (see for instance [23]). Here we are going to introduce a chemical potential $\mu$ into the Dirac equation.

The next ingredient of the construction is to take into account vortices generated by topological defects in the honeycomb lattice [24, 25, 26, 27]. To this end, we introduce $U(1) \times U(1)$ gauge fields $a_{\mu}$ and $b_{\mu}$ coupled with fermions, to be identified with the ordinary electromagnetic potential and the chiral gauge field, respectively [27, 23]. Thus the action of the resulting coupled system reads

$$
S\left[\psi, \bar{\psi} ; a_{\mu}, b_{\mu}\right]=-\kappa \int d^{3} x \bar{\psi}_{s}\left(i \gamma^{\mu} \partial_{\mu}-\gamma^{\mu} a_{\mu}-\gamma_{5} \gamma^{\mu} b_{\mu}-\mu \gamma_{0}\right) \psi^{s}
$$

where $\kappa$ is for the moment an arbitrary constant and we have included a further index $s=1,2$ which takes into account the (real) spin degeneracy of particles [28. It is worth noting that the form of this action resembles the one considered in [29] in connection with Topological Insulators. The final ingredient amounts to include properly the presence of (unavoidable) disorder on the graphene sheet [30, 31]. Following the argument of the authors of [29], we do not add an explicit disorder potential but rather exploit the replica method, a tool employed already in graphene monolayer, see [32, 33]. This means that we can introduce $N$ replicas in the action (3) where fermions are coupled with $U(N) \times U(N)$ gauge fields denoted by

$$
\mathbf{a}_{\mu}=a_{\mu}^{\alpha} T_{\alpha} ; \quad \mathbf{b}_{\mu}=b_{\mu}^{\alpha} T_{\alpha}
$$

where $T_{\alpha}$ are the (Hermitian) generators of $U(N)$. 


\section{Euclidean effective action}

The Euclidean (Wick rotated) counterpart of the action (3) written in terms of the fields (4) is obtained by performing the following substitutions

$$
t \rightarrow i \tau ; \quad \gamma^{\mu} \partial_{\mu} \rightarrow i \gamma^{\mu} \partial_{\mu} ; \quad \gamma^{\mu} \mathbf{a}_{\mu} \rightarrow i \gamma^{\mu} \mathbf{a}_{\mu} \quad \gamma^{\mu} \mathbf{b}_{\mu} \rightarrow i \gamma^{\mu} \mathbf{b}_{\mu}
$$

By integrating out the fermionic fields, the Euclidean effective action depends on the two gauge fields alone

$$
\int \mathcal{D}\left[\psi_{1}, \bar{\psi}_{1}, \psi_{2}, \bar{\psi}_{2}\right] e^{-S}=e^{-S_{\mathrm{eff}}\left[\mathbf{a}_{\mu}, \mathbf{b}_{\mu}\right]}
$$

The derivative expansion of $S_{\text {eff }}$ reads

$$
S_{\mathrm{eff}}=-\operatorname{Tr} \ln \left(G_{0}^{-1}\right)+\sum_{n=1}^{\infty} \frac{1}{n} \operatorname{Tr}\left[G_{0}\left(-i \gamma^{\mu} \mathbf{a}_{\mu}-i \gamma^{\mu} \mathbf{b}_{\mu}\right)\right]^{n},
$$

where $G_{0}$ is the propagator of free Dirac fermions.

At leading order and upon applying a Pauli-Villars regularization, we obtain

$$
\tilde{S}_{\text {eff }}=\kappa\left(\frac{2}{4 \pi} I\left[\mathbf{A}^{+}\right]-\frac{2}{4 \pi} I\left[\mathbf{A}^{-}\right]\right)
$$

where the $\mathbf{A}_{\mu}^{ \pm}$(in terms of anti-Hermitian generators) are related to the original gauge fields by

$$
\mathbf{A}_{\mu}^{ \pm}=\mathbf{a}_{\mu} \pm \mathbf{b}_{\mu}
$$

and where ( $\operatorname{tr}$ is the trace over Lie algebra labels and $\epsilon^{\mu \nu \sigma}$ is the Levi-Civita totally antisymmetric symbol)

$$
I\left[\mathbf{A}_{\mu}^{ \pm}\right]=\int d^{3} x \epsilon^{\mu \nu \sigma} \operatorname{tr}\left(\mathbf{A}_{\mu}^{ \pm} \partial_{\nu} \mathbf{A}_{\sigma}^{ \pm}+\frac{2}{3} \mathbf{A}_{\mu}^{ \pm} \mathbf{A}_{\nu}^{ \pm} \mathbf{A}_{\sigma}^{ \pm}\right) .
$$

Looking at the numerical constant inside the bracket in (8) and comparing it with the standard form of Chern-Simons action, $\tilde{S}_{\text {eff }}$ turns out to be proportional (trough $\kappa$ ) to a double non-Abelian $U(N)_{2} \times \overline{U(N)}_{2}$ CS at level $k=2$ (recall that the level represents the quantized CS coupling constant, an integer multiple of $(4 \pi)^{-1}$, and the overline means that the second Chern-Simons term has opposite chirality).

By resorting to results found in the 90's about the level-rank duality [34, 35], considered here in a CS environment, the exchange between the 
rank $N$ of the gauge group and the level $k$ provides a consistent dualized $U(2)_{N} \times \overline{U(2)}_{N}$ action. A dual model shares the same fusion rules, modular transformation matrices and observables of its parent theory, so that the two are to be considered as equivalent also in the present context of effective field theories, as pointed out in [13](Section III).

On applying to the partition function $Z$ associated with (88) the formal $N \rightarrow 0$ limit we get

$$
\begin{gathered}
\overline{S_{\text {eff }}}:=\left.\frac{d}{d N} Z^{N}\right|_{N=0}=\left.\kappa \frac{d}{d N} \frac{2}{4 \pi}\left\{I\left[\mathbf{A}^{+}\right]-I\left[\mathbf{A}^{-}\right]\right\}\right|_{N=0}= \\
\left.\kappa \frac{d}{d N} \frac{N}{4 \pi}\left\{I\left[\mathbf{A}^{+}\right]-I\left[\mathbf{A}^{-}\right]\right\}\right|_{N=0}=\frac{\kappa}{4 \pi}\left\{I\left[\mathbf{A}^{+}\right]-I\left[\mathbf{A}^{-}\right]\right\},
\end{gathered}
$$

where the switch $N \leftrightarrow k$ has been explicitly included.

The so far unrestricted effective coupling constant $\kappa$ is now required to assume only integer values according to the standard argument used whenever the Feynman quantization prescription is going to be carried out in a topological field theory background. Thus (11) represents a $U(2)_{\kappa} \times \overline{U(2)}_{\kappa}$ double CS action endowed with an 'effective level' $\kappa$. This topological field theory has been shown to be equivalent (both at the classical and at the quantum level) to a BF-type theory [11, 36, 37, 38] with a classical action given by

$$
S_{B F, \lambda}=\int d^{3} x \epsilon^{\mu \nu \sigma} \operatorname{tr}\left(\mathbf{B}_{\mu} \mathbf{F}_{\nu \sigma}+\frac{\lambda^{2}}{3} \mathbf{B}_{\mu} \mathbf{B}_{\nu} \mathbf{B}_{\sigma}\right)
$$

Here $\lambda$ is a constant, related to $\kappa$ by

$$
\lambda^{2}=\left(\frac{4 \pi}{\kappa}\right)^{2},
$$

$\mathbf{F}_{\nu \sigma}$ is the curvature 2-form associated with the connection 1-form $\mathbf{A}_{\mu}$ according to

$$
\mathbf{F}_{\nu \sigma}=\partial_{\nu} \mathbf{A}_{\sigma}-\partial_{\sigma} \mathbf{A}_{\nu}+\left[\mathbf{A}_{\nu}, \mathbf{A}_{\sigma}\right]
$$

$\mathbf{B}_{\mu}$ (the B-field) is canonically conjugate to $\mathbf{A}_{\mu}$ and

$$
\mathbf{A}_{\mu}=\frac{1}{2}\left(\mathbf{A}_{\mu}^{+}+\mathbf{A}_{\mu}^{-}\right) \quad \mathbf{B}_{\mu}=\frac{\kappa}{8 \pi}\left(\mathbf{A}_{\mu}^{+}-\mathbf{A}_{\mu}^{-}\right)
$$

The standard terminology 'BF action with a (positive) cosmological constant term $\lambda^{2}$, for the action (12) bears on the fact that, once chosen a 
3-dimensional, compact and oriented Riemannian manifold $M^{3}$, up to a suitable isomorphism this action would be mapped into the (first-order form of) Euclidean Einstein-Hilbert action of General Relativity (given by the first integral) plus a term proportional to the volume of the underlying spacetime $M^{3}$. The relevant gauge group would be $S O(3)$ (or its universal covering $S U(2))$ and the fields would have a geometric nature: the field strength $\mathbf{F}_{\nu \sigma}$ can be related to (a contraction of) the Riemann tensor and the B-field to the dreibein (an orthonormal set of three basis vectors expressed in suitable local coordinates).

\section{Observables}

Coming back to the interpretation of (12) as an effective action for graphene, the original gauge fields are related through (9) to the BF fields by

$$
\mathbf{a}_{\mu}=\mathbf{A}_{\mu} \quad \mathbf{b}_{\mu}=\frac{4 \pi}{\kappa} \mathbf{B}_{\mu} \equiv \lambda \mathbf{B}_{\mu}
$$

so that the $\mathrm{BF}$ framework complies with the description at the microscopic level. Thus the $\mathbf{A}$-field is consistently interpreted as the (non-Abelian counterpart of the) electromagnetic potential, while the 'chiral' $\mathbf{B}$-field bears on the presence of vortices. Geometrically the $\mathbf{A}$-field is still a connection, while the 1-form $\mathbf{B}$ is related to 1-dimensional submanifolds embedded into the 3dimensional background. (Note that the shift in front of $\mathbf{B}_{\mu}$ in (16) does not alter the nature of this field, a fact that would not be true for a connection 1 -form).

Quantum observables in a BF framework are found on applying the standard machinery of $3 \mathrm{~d}$ TQFT. First, the generating functional is formally given by the path integral

$$
Z_{B F, \lambda}\left[M^{3}\right]=\int \mathcal{D} \mathbf{A} \mathcal{D} \mathbf{B} \exp \left\{i S_{B F, \lambda}(\mathbf{A}, \mathbf{B})\right\}
$$

for a fixed background 3-manifold $M^{3}$ (the integration domain in the expression of $S_{B F, \lambda}$ in (12)). In a purely geometric, field theoretic context $M^{3}$ is a closed, oriented Riemannian manifold and it can be shown that the functional (17) is a topological invariant of the manifold, related to the square 
of the modulus of the Witten-Chern-Simons [38, 39, 36]. In the present context of $2 \mathrm{~d}$ graphene sheets embedded into a $(2+1)$-dimensional background suitable boundary (edge) terms should be taken into account explicitly.

Gauge invariant quantum observables are associated with embedded oriented (closed) curves $C \subset M^{3}$. More precisely [36], vacuum expectation values of observables in an $S U(N) \mathrm{BF}$ are given formally by

$$
Z_{B F, \lambda}\left[M^{3}, C\right]=\int \mathcal{D} \mathbf{A} \mathcal{D} \mathbf{B} \exp \left\{i S_{B F, \lambda}(\mathbf{A}, \mathbf{B})\right\} \operatorname{tr} \operatorname{Hol}(\mathbf{A} \pm \lambda \mathbf{B}),
$$

where $\operatorname{tr}$ is over Lie algebra labels and $\operatorname{Hol}(\mathbf{A} \pm \lambda \mathbf{B})$ are holonomies

$$
\operatorname{Hol}(\mathbf{A} \pm \lambda \mathbf{B})=\exp \left\{i \int_{C}\left(\mathbf{A}_{\mu} \pm \lambda \mathbf{B}_{\mu}\right) d z^{\mu}\right\}
$$

evaluated (up to path ordering) along the curve $C$ parametrized by local coordinated $z^{\mu}$.

We argue that the quantization of the $U(2)_{\kappa}$ BF setting described in the previous section will provide explicit expressions for quasi-particles excitations associated with closed paths surrounding vortices. Issues that should be worked out include the choice of boundary conditions, the selection of a proper gauge fixing and possibly perturbative expansions of observables in terms of powers of $(4 \pi / \kappa)=\lambda$ for $\lambda \rightarrow 0$. Work is in progress to improve these developments.

\section{Concluding remarks}

The detection of topological effects in graphene represents, on the one hand, a major challenge for graphene physics (possibly also in view of applications to topological quantum computing) and an ideal playground for testing geometrical models and methods [6], on the other. Looking in particular at the morphology of graphene, there have been observed non-planar arrangements of bent graphene sheets, carbon nanotubes, fullerenes and also schwarzites [40] which are respectively associated with cylindrical, spherical and hyperbolic configurations. Carbon nanocones [41] are associated with singular (i.e. not smooth) surfaces. The BF setting presented in this paper

seems particularly suitable to model also the effective behavior of samples of graphene sheets with different intrinsic geometry and equipped with a variety of boundary conditions. 
As mentioned in the introduction, the crucial feature that makes BFtheory so promising in the quantum computational context is given by the equivalence of BF quantum functionals with Turaev-Viro (TV) state sum models [39]. The latter provide an ab initio discretized and colored ambient 3 -manifolds possibly endowed with (piecewise linear) graphs or loops (the colorings on the $3 \mathrm{~d}$ triangulation and on 1-dimensional subsets are induced by elements of the $S U(2)_{\kappa}$ representation ring). Thus the continuous geometric picture outlined in the previous sections would become fully discretized, with functional integrals replaced by combinatorial, finite-type state functionals to be associated with ground states and (evolving) edge or point-like configurations carrying fractionary charges. The TV setting has been already addressed in connection with abstract models for topological quantum computation [42, 43, 44. A proper inclusion of the effective behavior of graphene in such an unified scheme would then represent a major theoretical achievement as well as a viable tool for explicit evaluations of significant physical quantities.

\section{Acknowledgments}

We are grateful to Giorgio Benedek, Giancarlo Jug, Zoltan Kádár and Silvano Garnerone for their comments. We are in debt with the referees for suggestions that have improved the presentation of the paper.

\section{References}

[1] Witten E. Commun. Math. Phys. 121 (1989) 351.

[2] Atiyah M. F. Publ. Math. IHES 68 (1989) 175.

[3] Wilczek F. Phys. Rev. Lett. 49 (1982) 957.

[4] Bernevig B. A. and Zhang S.-C. Phys. Rev. Lett.96(2006) 106802.

[5] Goldman N., Satija I., Bermudez A., Martin-Delgado M. A., Lewenstein M., Spielman I. B. Phys. Rev. Lett. 105(2010), 255302.

[6] Pachos J. K. Contemp. Phys. 50(2009), 375.

[7] Levin M. A., Wen X.-G. Phys. Rev. B. 71 (2005) 045110. 
[8] Kitaev A. AIP Conf. Proc. 1134 (2009) 22.

[9] Nayak C., Simon S. H., Stern A., Freedman M. H., Das Sarma S. Rev. Mod. Phys. 80 (2008) 1083.

[10] Freedman M. H., Kitaev A., Larsen M. J., Wang Z. arXiv:quant-ph/0101025 (2002).

[11] Birmingham D., Blau M., Rakowski M, Thomson G. Phys. Rep. 209 (1991) 129.

[12] Kaul R. K., Govindarajan T. R., Ramadevi P. arXiv:hep-th/0504100(2005).

[13] Freedman M., Nayak C., Shtengel K., Walker K., Wang Z. Ann. Phys.310 (2004)428.

[14] Cho G. Y., Moore J. E. Ann. Phys. 326 (2011) 1515.

[15] Blasi A., Braggio A., Carrega M., Ferraro D., Maggiore N., Magnoli N. arXiv:1106.4641v2 (2011).

[16] Ardonne E., Fendley P., Fradkin E. Ann. Phys. 310 (2004) 493.

[17] Fendley P., Fradkin E. Phys. Rev. B 72 (2005) 024412.

[18] Hansson T. H., Oganesyan V., Sondhi S. L. Ann. Phys. 313(2004) 497.

[19] Novoselov K. S., Geim A. K., Morozov S. V., Jiang D., Zhang Y., Dubonos S. V., Grigorieva I. V., Firsov A. A. Science 306 (2004) 666.

[20] Beenakker C. W. J. Rev. Mod. Phys. 80 (2008) 1337.

[21] Castro Neto A. H. Material Today 13 (2010) 12.

[22] Semenoff G. W. Phys. Rev. Lett. 53 (1984) 2449.

[23] Ryu S., Mudry C., Hou C.-Y., Chamon C. Phys. Rev. B 80 (2009) 205319.

[24] Vozmediano M. A. H., Katsnelson M. I., Guinea F. Phys. Rep. 496 (2010) 109. 
[25] Jackiw R., Pi S.-Y. Phys. Rev. Lett. 98 (2007) 266402.

[26] Seradjeh B., Franz M. Phys. Rev. Lett. 101 (2008) 146401.

[27] Hou C.-Y., Chamon C., Mudry C. Phys. Rev. Lett. 98 (2007) 186809.

[28] Son D. T. Phys. Rev. B 75 (2007) 235423.

[29] Schnyder A. P., Ryu S., Furusaki A., Ludwig A. W. W. Phys. Rev. B 78 (2008) 195125.

[30] Pereira V. M., Lopes dos Santos J. M. B., Castro Neto A. H. Phys. Rev. B 77 (2008) 115109.

[31] Ostrovsky P. M., Gornyi I.V., Mirlin A. D. Phys. rev. B 74 (2006) 235443

[32] Altland A. Phys. Rev. Lett. 97 (2006) 236802.

[33] Sinner A., Sedrakyan A., Ziegler K. Phys. Rev. B 83 (2011) 155115.

[34] Naculich S. G., Riggs H. A., Schnitzer H. J. Phys. Lett. B 246 (1990) 417.

[35] Mlawer E. J., Naculich S. G., Riggs H. A., Schnitzer H. J. Nucl. Phys. B 352 (1991) 863.

[36] Cattaneo A. S., Cotta-Ramusino P., Fröhlich J., Martellini M. J. Math. Phys. 36 (1995) 6137.

[37] Archer F., Williams R. M. Phys. Lett. B 273 (1991) 438.

[38] Witten E. Nucl. Phys. B 311 (1988) 46.

[39] Turaev V.G. Quantum Invariants of Knots and 3-Manifolds W. de Gruyter, Berlin (1994)

[40] Spagnolatti I., Bernasconi M., Benedek G. Eur. Phys. J. B 32 (2003) 181.

[41] Heiberg-Andersena H., Skjeltorp A. T., Sattler K. J. Non-Cryst. Solids 354 (2008) 524

[42] Kádár Z., Marzuoli A., Rasetti M. Int.J.Quant.Inf. 7 (2009) 195. 
[43] Kádár Z., Marzuoli A., Rasetti M. Adv. Math. Phys. 2010 (2010) 671039 .

[44] Koenig R., Kuperberg G., Reichardt B. W. Ann. Phys. bf 325 (2010) 2707. 